\title{
Metallic abundances of the 2002 Leonid meteor deduced from high-definition TV spectra
}

\author{
T. Kasuga ${ }^{1,4}$, T. Yamamoto ${ }^{2,3}$, J. Watanabe ${ }^{4}$, N. Ebizuka ${ }^{5}$, H. Kawakita ${ }^{6}$, and H. Yano ${ }^{7}$ \\ 1 Department of Astronomical Science, School of Physical Science, The Graduate University for Advanced Studies, \\ 2-21-1 Osawa, Mitaka, Tokyo 181-8588, Japan \\ e-mail: kasugats@cc.nao.ac.jp \\ 2 Institute of Low Temperature Science, Department of Earth and Planetary Sciences, Hokkaido University, Kita-19, Nishi-8, \\ Kita-ku, Sapporo, Hokkaido 060-0819, Japan \\ 3 Department of Earth and Planetary Sciences, Nagoya University, Nagoya 464-8602, Japan \\ e-mail: ty@lowtem.hokudai.ac.jp \\ 4 National Astronomical Observatory of the Japan (NAOJ), National Institute of Natural Science, 2-21-1 Osawa, Mitaka, \\ Tokyo 181-8588, Japan \\ e-mail: jun.watanabe@nao.ac.jp \\ 5 V-CAD High Speed Computer System Team, The Institute of Physical and Chemical Research (RIKEN), Wako, \\ Saitama 351-0198, Japan \\ e-mail: ebizuka@riken.go.jp \\ ${ }^{6}$ Gunma Astronomical Observatory, 6860-86 Nakayama, Takayama, Agatsuma, Gunma 377-0702, Japan \\ e-mail: kawakita@astron.pref.gunma.jp \\ 7 Institute of Space and Astronautical Science (ISAS), Japan Aerospace Exploration Agency (JAXA), 3-1-1 Yoshinodai, \\ Sagamihara, Kanagawa 229-8510, Japan \\ e-mail: yano.hajime@jaxa.jp
}

Received 14 August 2003 / Accepted 28 December 2004

\begin{abstract}
High-definition TV spectra in the ultraviolet-visible region were obtained during the 2002 Leonid aircraft campaign. We analyze the spectra of the brightest fireball that appeared at $03^{\mathrm{h}} 47^{\mathrm{m}} 54^{\mathrm{s}}$ UT on Nov. 19, 2002 and identify the neutral atoms, mainly MgI, FeI, CaI, and NaI in the observed wavelengths between $300-650 \mathrm{~nm}$. The singly ionized atomic emissions, CaII and MgII lines, also appeared in the spectrum in several epochs during the series of video frames. From analysis of the spectra, time variation in the abundances of metallic atoms, along with their electronic excitation and blackbody temperatures, were obtained assuming the Local Thermal Equilibrium (LTE) condition. Both Fe and $\mathrm{Ca}$ abundances relative to $\mathrm{Mg}$ are lower than the solar abundance, while $\mathrm{Na}$ is slightly higher. We found correlation between the excitation temperature and the abundance of $\mathrm{Ca}$, which suggests incomplete evaporation of the Ca due to intrinsic refractoriness. A search for bands of CHON-related molecules, such as $\mathrm{OH}$ and $\mathrm{CN}$, is not successful in the brightest fireball in this study.
\end{abstract}

Key words. meteors, meteroids

\section{Introduction}

Meteors represent one sign of meteoroids falling to Earth from space and constantly bringing extraterrestrial matter with them. Influx is thought to have been much greater at an early stage of our solar system than it is at present. Meteoroids are also regarded as candidates for carrying prebiotic organic matter contributing to the origin of life, so that more knowledge about both the constitution of meteoroids and their alteration during ablation offer keys to the early molecular evolution of our Earth (Jenniskens et al. 2000a).

Although spectroscopy is the usual method for probing the chemical composition of astronomical bodies, it is difficult to apply to meteors for two reasons. One is their randomness, such that we can predict neither their positions nor the times they will appear. This makes it difficult to perform high quality spectroscopic observation of meteors. Another reason is the short duration of meteor illuminators, which is caused by violent ablation during its encounter with the upper atmosphere. There is nothing more to do than to await a bright meteor's appearance inside the instrument's small field of view (FOV), meaning that spectroscopic observation remains a matter of chance alone. Hence, the spectroscopic observations of the meteors depends on "luck".

The probability of indeed observing them successfully increases dramatically during high flux meteor showers or storms. The latest activities of the Leonid meteor showers 
associated with passage of the parent comet 55P/Tempel-Tuttle provided ideal conditions for observing these meteors. After the return of the parent comet in 1998, several theoretical calculations predicted that the peak activities could reach storm levels in the next five years (McNaught \& Asher 1999, 2001). The Leonids are the fastest $\left(72 \mathrm{~km} \mathrm{~s}^{-1}\right)$ of all meteor showers to enter the Earth's atmosphere, because Comet 55P/TempelTuttle has a retrograde orbit with respect to the Earth's heliocentric motion. This results in the Leonid meteors reserving the highest import energy into the atmosphere; thus the excitation flash from ablation can be observed more easily than with other meteor showers. This rare occasion to improve on our data for meteors led to several world-wide campaigns being organized around the appearance of the Leonids in 19982002. Among these, the Leonid Multi-Instrument Aircraft campaign which had started in 1998 (Leonid MAC) has brought the greatest advance in meteor astronomy (Jenniskens 2003; Jenniskens \& Butow 1999; Jenniskens et al. 2000b). This mission's goal is to bring together scientists in different disciplines and from all over the world to cooperate on observations of the Leonid meteors using wide ranging techniques. These onboard techniques are carried out in airplanes at $\sim 10 \mathrm{~km}$ altitude under guaranteed clear weather conditions.

Since 2001, we have been focusing on spectroscopic observations of the Leonid meteors in the ultraviolet region, where bands of CHON - related molecules such as $\mathrm{OH}$ and $\mathrm{CN}$ are expected, as well as many lines of metallic atoms. We have participated in the Leonid MAC mission (Jenniskens 2002a) since 1998 and developed the High-Definition TV (HDTV) spectroscopic observational system focused on the near ultraviolet wavelength range, which enabled us to reduce much air extinction and Rayleigh scattering in the ultraviolet region of the Leonid meteor. The HDTV video spectroscopy also enabled us to obtain higher time resolution for meteor spectra data (i.e., time resolution of HDTV is $0.033 \mathrm{~s}$ ). In turn, this has enabled us to study the more detailed time variation in metallic abundances, excitation temperature, and blackbody temperature. In this paper, we describe results from analysis of representative spectra of the Leonid meteor taken by the airborne HDTV system on 19 November 2002.

\section{Observation}

The HDTV ultraviolet spectroscopic observation was performed onboard a NASA DC-8 airplane during the 2002 Leonid MAC mission. At the same time, TV observation was also performed onboard the Flying Infrared Signature Technology Aircraft (FISTA). Two aircraft were used to determine the altitude of the meteors. The flight route was from Torrejon, Spain, to Offutt, Nebraska, USA in order to be certain to detect both two predicted peaks (Lyytinen \& Van Flandern 2000; McNaught \& Asher 2002; Jenniskens 2002b; Vaubaillon 2002).

The HDTV spectroscopic observational system sensitive to UV - visible (in $250-700 \mathrm{~nm}$ ) consists of the reflective grating (600 grooves $/ \mathrm{mm}$, blaze $300 \mathrm{~nm})$, the UV lens $(f=30 \mathrm{~mm}$, F1.4), the Image Intensifier (I.I.) sensitive to UV - visible, and the HDTV camera, while an HDTV camera has been used for

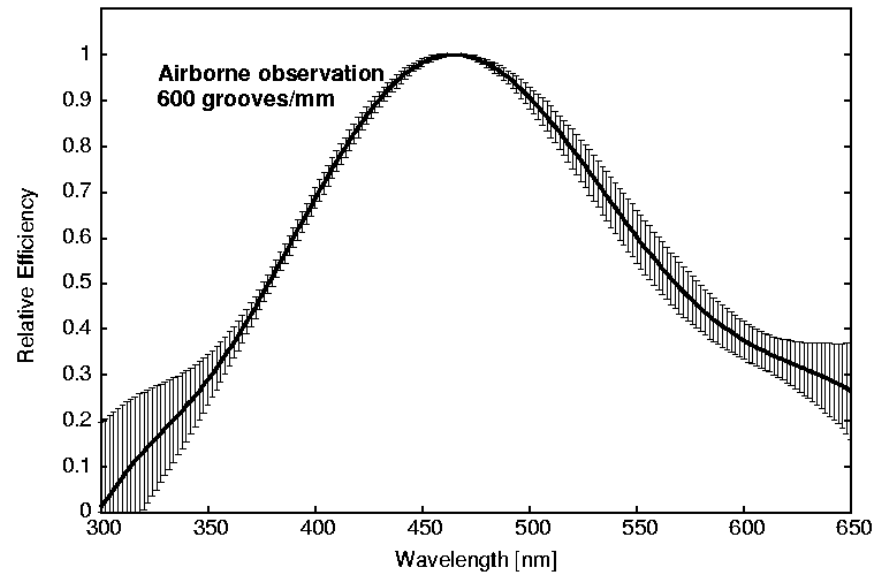

Fig. 1. The normalized system efficiencies (NSEs). The solid curve indicates the NSE of the HDTV spectroscopic observational system onboard with the grating of 600 grooves $/ \mathrm{mm}$ (blaze $300 \mathrm{~nm}$ ), calibrated with Jupiter.

meteor imaging since 1998 (Watanabe et al. 1999). The 1-inch 2M pixel FIT CCD of the HDTV has resolution as high as 1150 TV lines, and the meteors were recorded as 8-bit images. The diagonal coverage of the FOV was $30^{\circ}$, and the observable bands were in $300-650 \mathrm{~nm}$. The maximum spectral resolution of $\sim 1.0 \mathrm{~nm}(\lambda / \Delta \lambda \sim 300)$ was achieved for the reflective grating mentioned above.

Figure 1 shows the system efficiency of airborne observation using the 600 grooves/mm grating. We used Jupiter as the calibration source. Here, the system efficiency $f(\lambda)$ is

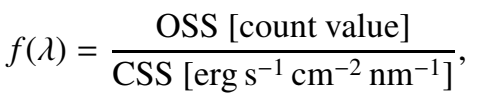

where OSS [count value] is observed Jovian spectrum data, and CSS[erg s-1 $\mathrm{cm}^{-2} \mathrm{~nm}^{-1}$ ] is Jovian data from the catalog. The CSS data were obtained by multiplication of the solar spectrum by the methane spectrum of Jupiter. (Wehrli 1985, AM0 Spectrum (http://rredc.nrel.gov/solar/standards/ am0/wehrli85.txt), Karkoschka 1994). The pixel interval of observed Jovian spectrum data, including the 0th, was corrected to wavelength interval using that of the meteor spectrum.

The efficiency of the HDTV spectroscopic observation system is affected by both air extinction and instrumental efficiency. The system efficiencies shown in Fig. 1 are normalized to unity at their maxima. Especially, efficiency at less than the $350 \mathrm{~nm}$ wavelength range, has large error bars because the flux of the Jovian ultraviolet reflect emission was very weak, although the observational system was intrinsically sensitive in the ultraviolet range (Kasuga et al. 2003).

Flux density $F$ of the meteor spectra was derived using efficiency curve $f(\lambda)$

$\frac{F(\lambda)}{\operatorname{erg~s}^{-1} \mathrm{~cm}^{-2} \mathrm{~nm}^{-1}}=\frac{\text { OMS [count value] }}{f(\lambda)}$

where OMS [count value] is the observed meteor spectrum data. 


\section{Analysis}

\subsection{Line identification}

During the Leonid MAC 2002 mission, the Japanese team obtained spectra of many Leonid meteors. In this paper, we focus on one spectrum obtained at $03^{\mathrm{h}} 47^{\mathrm{m}} 54^{\mathrm{s}}$ UT Nov. 19 , which is one of the highest quality data sets from the first peak activity of the 2002 Leonids. Altitude at the beginning of the meteor illuminations was estimated to be about $110 \mathrm{~km}$, owing to onset of the [OI] $(557 \mathrm{~nm})$ emission (Millman et al. 1971). The NASA DC -8 aircraft's position was latitude $+48.062^{\circ} \mathrm{N}$, longitude $17.427^{\circ} \mathrm{W}$, and altitude $8535 \mathrm{~m}$ as measured by the GPS system. Figure 2 shows time variation of the spectra at $03^{\mathrm{h}} 47^{\mathrm{m}} 54^{\mathrm{s}}$ UT ranging from 54.033 to $54.363 \mathrm{~s}$ at a time interval of $0.033 \mathrm{~s}$. Emissions around $300 \mathrm{~nm}$ were out of the FOV before $0.165 \mathrm{~s}$.

At wavelengths longer than $600 \mathrm{~nm}$, most of the emission features originated from the Earth's atmosphere, whereas most of the features below $600 \mathrm{~nm}$ originate in the meteor. Many lines of metallic elements appear which may be identified by the line catalog as shown in Table 1. This list includes the elements and their line positions in the observed wavelength regions, together with the Einstein $A$ coefficient, energy levels $E_{1}$ and $E_{\mathrm{u}}$ of the lower and upper levels, configurations, and the statical weights $g_{1}$ and $g_{\mathrm{u}}$ of the lower and upper levels. We considered all the transitions listed in Table 1 for identification, a process then applied to each spectrum shown in Fig. 2.

Figure 3 shows an example of line identification for the spectrum at $0.165 \mathrm{~s}$, when the emissions around $300 \mathrm{~nm}$ came into its own FOV. Under $350 \mathrm{~nm}$ and over $625 \mathrm{~nm}$ in wavelength, errors tend to be large due to the poor efficiency at the wavelength regions shown in Fig. 1. In the near ultraviolet region around $300-400 \mathrm{~nm}$, bands of CHON-related molecules, such as $\mathrm{OH}$ and $\mathrm{CN}$, are expected, as well as many lines of metallic atoms. In the lines near $309 \mathrm{~nm}$, bands of interesting molecules, such as $\mathrm{OH} A^{2} \Sigma^{+}-X^{2} \Pi(0-0)$, were observed in another Leonid meteor in a different year (Jenniskens et al. 2002). However, we could not detect any emissions related to the $\mathrm{OH}$ and $\mathrm{CN}$ because of poor efficiency. The observed meteor spectrum, as shown in the thick line, was obtained by subtracted blackbody. Metallic atom emission lines at $518 \mathrm{~nm}$ (Mg-triplet) and $589 \mathrm{~nm}$ (Na-doublet) are lines typical of the Leonid meteor. The lines at $358 \mathrm{~nm}(\mathrm{FeI}), 374 \mathrm{~nm}(\mathrm{FeI}), 383 \mathrm{~nm}$ (MgI, FeI), $404 \mathrm{~nm}(\mathrm{FeI}), 423 \mathrm{~nm}(\mathrm{CaI})$, and $438 \mathrm{~nm}$ (FeI) were also identified. The lines at around $393-396 \mathrm{~nm}$ and $448 \mathrm{~nm}$ are recognized during whole the meteor emission series. These lines can be interpreted as the ionized emissions of $\operatorname{CaII}(393,396)$ and $\mathrm{MgII}$. The former emission lines should have been observed as if they were one line, because they are too close to be resolved due to low spectral resolution. However, these lines may be due to neutral atoms, such as FeI at an early emission phase. This problem will be discussed later in Sect. 4.2.

The dotted line indicates the best fit model by neutral atomic emissions as is described in the next subsection. These line ratios are slightly varied for each frame, which allows us to have more detailed information about variations in meteor composition, along with the time series of meteor emission.

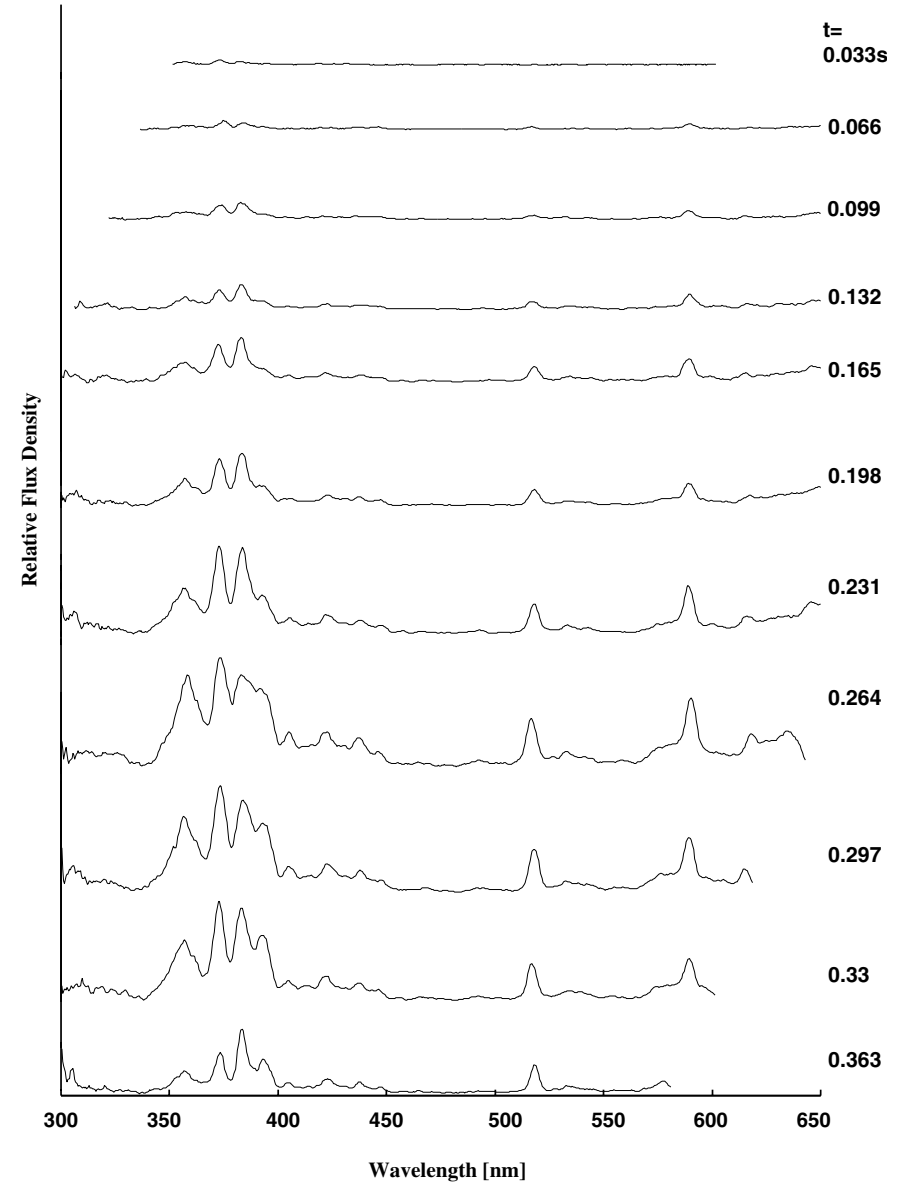

Fig. 2. Time variation of the spectra of the representative Leonid meteor observed at $03^{\mathrm{h}} 47^{\mathrm{m}} 54^{\mathrm{s}}$ UT on November 19,2002 . The time interval is $0.033 \mathrm{~s}$. The emissions around $300 \mathrm{~nm}$ were out of the field of view before $0.165 \mathrm{~s}$.

\subsection{Abundances of metallic elements}

For the following calculation, we assume that the meteor is a sphere of uniform brightness. The blackbody radiation caused by the heated surface of the meteoroid was recognized in the observed wavelength range. We fitted the blackbody continuum to the baseline of the observed spectrum, especially at $450-485 \mathrm{~nm}$ for each frame, and subtracted it from the observed spectrum (Borovička 1999). The blackbody radiation by a spherical meteoroid plasma is expressed as

$B(\lambda)=\pi \cdot \frac{2 h c^{2}}{\lambda^{5}}\left[\frac{1}{\exp \left(\frac{h c}{k_{\mathrm{B}} \widehat{T}_{\mathrm{e}} \lambda}\right)-1}\right] \quad\left[\operatorname{erg~s}^{-1} \mathrm{~cm}^{-2} \mathrm{~nm}^{-1}\right]$,

where $\widetilde{T}_{\mathrm{e}}$ is the blackbody temperature, $h$ Plank's constant, $c$ the velocity of light, $k_{\mathrm{B}}$ Boltzmann's constant, and $\lambda$ the wavelength.

In this study, we assume Local Thermal Equilibrium (LTE) for the population of each energy level; its validity will be examined later. Then, a total number of neutral atoms $N_{\mathrm{u}}$ in the upper energy level $E_{\mathrm{u}}$ is expressed as

$N_{\mathrm{u}}=\frac{g_{\mathrm{u}}}{g_{0}} N_{0} \exp \left(-\frac{E_{\mathrm{u}}}{k_{\mathrm{B}} T_{\mathrm{e}}}\right)$ 
Table 1. Metallic lines in the visible wavelength.

\begin{tabular}{|c|c|c|c|c|c|}
\hline Observed element & Catalog [nm] & $A_{\mathrm{ki}} / 10^{8} \mathrm{~s}^{-1}$ & $E_{1}-E_{\mathrm{u}}$ & Configurations & $g_{1}-g_{\mathrm{u}}$ \\
\hline $356 \mathrm{Fe} \mathrm{I}$ & 356.5379 & $3.8 \mathrm{e}-01$ & $0.958157-4.434614$ & $3 d 7(4 F) 4 s-3 d 7(4 F) 4 p$ & $7-9$ \\
\hline $357 \mathrm{Fe} \mathrm{I}$ & 357.0097 & $6.77 \mathrm{e}-01$ & $0.914602-4.386464$ & $3 d 7(4 F) 4 s-3 d 7(4 F) 4 p$ & $9-11$ \\
\hline${ }^{1} 358 \mathrm{Fe} \mathrm{I}$ & 358.1195 & $1.02 \mathrm{e}+00$ & $0.858995-4.320100$ & $3 d 7(4 F) 4 s-3 d 7(4 F) 4 p$ & $11-13$ \\
\hline $371 \mathrm{Fe} \mathrm{I}$ & 371.9935 & $1.62 \mathrm{e}-01$ & $0.000000-3.332020$ & $3 \mathrm{~d} 6.4 \mathrm{~s} 2-3 \mathrm{~d} 6(5 \mathrm{D}) 4 \mathrm{~s} .4 \mathrm{p}\left(3 \mathrm{P}^{*}\right)$ & $9-11$ \\
\hline \multirow[t]{2}{*}{$372 \mathrm{Fe} \mathrm{I}$} & 372.2563 & $4.97 \mathrm{e}-02$ & $0.087285-3.416953$ & $3 \mathrm{~d} 6.4 \mathrm{~s} 2-3 \mathrm{~d} 6(5 \mathrm{D}) 4 \mathrm{~s} .4 \mathrm{p}(3 \mathrm{P} *)$ & $5-5$ \\
\hline & 372.7619 & $2.25 \mathrm{e}-01$ & $0.958157-4.283309$ & $3 d 7(4 F) 4 s-3 d 7(4 F) 4 p$ & $7-5$ \\
\hline \multirow[t]{3}{*}{$373 \mathrm{Fe} \mathrm{I}$} & 373.3317 & $6.2 \mathrm{e}-02$ & $0.110114-3.430191$ & $3 \mathrm{~d} 6.4 \mathrm{~s} 2-3 \mathrm{~d} 6(5 \mathrm{D}) 4 \mathrm{~s} .4 \mathrm{p}\left(3 \mathrm{P}^{*}\right)$ & $3-3$ \\
\hline & 373.4864 & $9.02 \mathrm{e}-01$ & $0.858995-4.177697$ & $3 d 7(4 F) 4 s-3 d 7(4 F) 4 p$ & $11-11$ \\
\hline & 373.7131 & $1.42 \mathrm{e}-01$ & $0.051569-3.368257$ & $3 \mathrm{~d} 6.4 \mathrm{~s} 2-3 \mathrm{~d} 6(5 \mathrm{D}) 4 \mathrm{~s} .4 \mathrm{p}(3 \mathrm{P} *)$ & $7-9$ \\
\hline \multirow[t]{5}{*}{$374 \mathrm{Fe} \mathrm{I}$} & 374.3362 & $2.60 \mathrm{e}-01$ & $0.990110-4.301279$ & $3 d 7(4 F) 4 s-3 d 7(4 F) 4 p$ & $5-3$ \\
\hline & 374.5561 & $1.15 \mathrm{e}-01$ & $0.087285-3.396509$ & $3 \mathrm{~d} 6.4 \mathrm{~s} 2-3 \mathrm{~d} 6(5 \mathrm{D}) 4 \mathrm{~s} .4 \mathrm{p}\left(3 \mathrm{P}^{*}\right)$ & $5-7$ \\
\hline & 374.5899 & $7.33 e-02$ & $0.121265-3.430191$ & $3 \mathrm{~d} 6.4 \mathrm{~s} 2-3 \mathrm{~d} 6(5 \mathrm{D}) 4 \mathrm{~s} .4 \mathrm{p}(3 \mathrm{P} *)$ & $1-3$ \\
\hline & 374.8262 & $9.15 e-02$ & $0.110114-3.416953$ & $3 \mathrm{~d} 6.4 \mathrm{~s} 2-3 \mathrm{~d} 6(5 \mathrm{D}) 4 \mathrm{~s} .4 \mathrm{p}(3 \mathrm{P} *)$ & $3-5$ \\
\hline & 374.9485 & $7.64 \mathrm{e}-01$ & $0.914602-4.220363$ & $3 d 7(4 F) 4 s-3 d 7(4 F) 4 p$ & 9-9 \\
\hline $375 \mathrm{Fe} \mathrm{I}$ & 375.8233 & $6.34 \mathrm{e}-01$ & $0.958157-4.256224$ & $3 d 7(4 F) 4 s-3 d 7(4 F) 4 p$ & $7-7$ \\
\hline \multirow[t]{2}{*}{$376 \mathrm{Fe} \mathrm{I}$} & 376.3789 & $5.44 \mathrm{e}-01$ & $0.990110-4.283309$ & $3 d 7(4 F) 4 s-3 d 7(4 F) 4 p$ & $5-5$ \\
\hline & 376.7191 & $6.40 \mathrm{e}-01$ & $1.011055-4.301279$ & $3 d 7(4 F) 4 s-3 d 7(4 F) 4 p$ & $3-3$ \\
\hline $378 \mathrm{FeI}$ & 378.6676 & $2.77 \mathrm{e}-02$ & $1.011055-4.284350$ & $3 \mathrm{~d} 7(4 \mathrm{~F}) 4 \mathrm{~s}-3 \mathrm{~d} 6(5 \mathrm{D}) 4 \mathrm{~s} .4 \mathrm{p}\left(3 \mathrm{P}^{*}\right)$ & $3-1$ \\
\hline $381 \mathrm{Fe} \mathrm{I}$ & 381.5840 & $1.3 e+00$ & $1.484864-4.733141$ & $3 d 7(4 \mathrm{~F}) 4 \mathrm{~s}-3 \mathrm{~d} 7(4 \mathrm{~F}) 4 \mathrm{p}$ & $9-7$ \\
\hline \multirow[t]{4}{*}{$382 \mathrm{Fe} \mathrm{I}$} & 382.0425 & $6.68 \mathrm{e}-01$ & $0.858995-4.103374$ & $3 d 7(4 F) 4 s-3 d 7(4 F) 4 p$ & $11-9$ \\
\hline & 382.4444 & $2.83 \mathrm{e}-02$ & $0.000000-3.240970$ & $3 \mathrm{~d} 6.4 \mathrm{~s} 2-3 \mathrm{~d} 6(5 \mathrm{D}) 4 \mathrm{~s} .4 \mathrm{p}(3 \mathrm{P} *)$ & $9-7$ \\
\hline & 382.5880 & $5.98 \mathrm{e}-01$ & $0.914602-4.154354$ & $3 d 7(4 F) 4 s-3 d 7(4 F) 4 p$ & $9-7$ \\
\hline & 382.7823 & $1.05 \mathrm{e}+00$ & $1.557357-4.795466$ & $3 d 7(4 F) 4 s-3 d 7(4 F) 4 p$ & $7-5$ \\
\hline $383 \mathrm{Fe} \mathrm{I}$ & 383.4222 & $4.53 \mathrm{e}-01$ & $0.958157-4.190861$ & $3 d 7(4 F) 4 s-3 d 7(4 F) 4 p$ & $7-5$ \\
\hline \multirow[t]{6}{*}{$383 \mathrm{Mg} \mathrm{I}$} & 382.93547 & $9.40 \mathrm{e}-01$ & $2.709105-5.945919$ & $3 \mathrm{~s} .3 \mathrm{p}-3 \mathrm{~s} .3 \mathrm{~d}$ & $1-3$ \\
\hline & 383.22993 & $7.03 e-01$ & $2.711592-5.945919$ & $3 \mathrm{~s} .3 \mathrm{p}-3 \mathrm{~s} .3 \mathrm{~d}$ & $3-3$ \\
\hline & 383.23039 & $1.27 \mathrm{e}+00$ & $2.711592-5.945915$ & $3 \mathrm{~s} .3 \mathrm{p}-3 \mathrm{~s} .3 \mathrm{~d}$ & $3-5$ \\
\hline & 383.8290 & $4.7 \mathrm{e}-02$ & $2.716640-5.945919$ & $3 s .3 p-3 s .3 d$ & $5-3$ \\
\hline & 383.82919 & $1.68 \mathrm{e}+00$ & $2.716640-5.945917$ & $3 \mathrm{~s} .3 \mathrm{p}-3 \mathrm{~s} .3 \mathrm{~d}$ & $5-7$ \\
\hline & 383.82946 & $4.20 \mathrm{e}-01$ & $2.716640-5.945915$ & $3 \mathrm{~s} .3 \mathrm{p}-3 \mathrm{~s} .3 \mathrm{~d}$ & $5-5$ \\
\hline \multirow[t]{3}{*}{$384 \mathrm{Fe} \mathrm{I}$} & 384.0437 & $4.70 \mathrm{e}-01$ & $0.990110-4.217584$ & $3 d 7(4 F) 4 s-3 d 7(4 F) 4 p$ & $5-3$ \\
\hline & 384.1048 & $1.3 e+00$ & $1.607895-4.834856$ & $3 \mathrm{~d} 7(4 \mathrm{~F}) 4 \mathrm{~s}-3 \mathrm{~d} 7(4 \mathrm{~F}) 4 \mathrm{p}$ & $5-3$ \\
\hline & 384.9969 & $6.06 \mathrm{e}-01$ & $1.011055-4.230538$ & $3 d 7(4 F) 4 s-3 d 7(4 F) 4 p$ & $3-1$ \\
\hline \multirow[t]{2}{*}{$385 \mathrm{Fe} \mathrm{I}$} & 385.6372 & $4.64 \mathrm{e}-02$ & $0.051569-3.265706$ & $3 \mathrm{~d} 6.4 \mathrm{~s} 2-3 \mathrm{~d} 6(5 \mathrm{D}) 4 \mathrm{~s} .4 \mathrm{p}(3 \mathrm{P} *)$ & $7-5$ \\
\hline & 385.9911 & $9.70 \mathrm{e}-02$ & $0.000000-3.211190$ & $3 \mathrm{~d} 6.4 \mathrm{~s} 2-3 \mathrm{~d} 6(5 \mathrm{D}) 4 \mathrm{~s} .4 \mathrm{p}(3 \mathrm{P} *)$ & 9-9 \\
\hline $386 \mathrm{Fe} \mathrm{I}$ & 386.5523 & $1.55 \mathrm{e}-01$ & $1.011055-4.217584$ & $3 \mathrm{~d} 7(4 \mathrm{~F}) 4 \mathrm{~s}-3 \mathrm{~d} 7(4 \mathrm{~F}) 4 \mathrm{p}$ & $3-3$ \\
\hline \multirow[t]{3}{*}{$387 \mathrm{Fe} \mathrm{I}$} & 387.2501 & $1.05 \mathrm{e}-01$ & $0.990110-4.190861$ & $3 d 7(4 F) 4 s-3 d 7(4 F) 4 p$ & $5-5$ \\
\hline & 387.8018 & $7.72 \mathrm{e}-02$ & $0.958157-4.154354$ & $3 d 7(4 F) 4 s-3 d 7(4 F) 4 p$ & $7-7$ \\
\hline & 387.8573 & $6.6 e-02$ & $0.087285-3.283025$ & $3 \mathrm{~d} 6.4 \mathrm{~s} 2-3 \mathrm{~d} 6(5 \mathrm{D}) 4 \mathrm{~s} .4 \mathrm{p}(3 \mathrm{P} *)$ & $5-3$ \\
\hline \multirow[t]{2}{*}{ Ca II } & 393.3663 & $1.47 \mathrm{e}+00$ & $0.000000-3.150985$ & $3 p 6(1 S) 4 s-3 p 6(1 S) 4 p$ & $2-4$ \\
\hline & 396.8469 & $1.4 \mathrm{e}+00$ & $0.000000-3.123350$ & $3 \mathrm{p} 6(1 \mathrm{~S}) 4 \mathrm{~s}-3 \mathrm{p} 6(1 \mathrm{~S}) 4 \mathrm{p}$ & $2-2$ \\
\hline Fe I & 404.5813 & $8.63 \mathrm{e}-01$ & $1.484864-4.548506$ & $3 \mathrm{~d} 7(4 \mathrm{~F}) 4 \mathrm{~s}-3 \mathrm{~d} 7(4 \mathrm{~F}) 4 \mathrm{p}$ & $9-9$ \\
\hline $\mathrm{Fe} \mathrm{I}$ & 406.3594 & $6.8 \mathrm{e}-01$ & $1.557357-4.607594$ & $3 d 7(4 F) 4 s-3 d 7(4 F) 4 p$ & $7-7$ \\
\hline $\mathrm{Ca} \mathrm{I}$ & 422.6728 & $2.18 \mathrm{e}+00$ & $0.000000-2.932513$ & $4 \mathrm{~s} 2-4 \mathrm{~s} .4 \mathrm{p}$ & $1-3$ \\
\hline Fe I & 438.3544 & $5.00 \mathrm{e}-01$ & $1.484864-4.312471$ & $3 \mathrm{~d} 7(4 \mathrm{~F}) 4 \mathrm{~s}-3 \mathrm{~d} 7(4 \mathrm{~F}) 4 \mathrm{p}$ & $9-11$ \\
\hline $\mathrm{Fe} \mathrm{I}$ & 440.4750 & $2.75 \mathrm{e}-01$ & $1.557357-4.371352$ & $3 d 7(4 F) 4 s-3 d 7(4 F) 4 p$ & $7-9$ \\
\hline \multirow[t]{3}{*}{$\mathrm{Mg}$ II } & 448.1126 & $2.23 \mathrm{e}+00$ & $8.863657-11.62969$ & $3 d-4 f$ & $6-8$ \\
\hline & 448.1150 & $1.49 \mathrm{e}-01$ & $8.863657-11.62967$ & $3 d-4 f$ & $6-6$ \\
\hline & 448.1325 & $2.08 \mathrm{e}+00$ & $8.863765-11.62967$ & $3 d-4 f$ & $4-6$ \\
\hline \multirow{3}{*}{$\begin{array}{c}518 \mathrm{Mg} \mathrm{I} \\
\text { (triplet) }\end{array}$} & 516.73213 & $1.16 \mathrm{e}-01$ & $2.709105-5.107828$ & $3 \mathrm{~s} .3 \mathrm{p}-3 \mathrm{~s} .4 \mathrm{~s}$ & $1-3$ \\
\hline & 517.26844 & $3.46 \mathrm{e}-01$ & $2.711592-5.107828$ & $3 \mathrm{~s} .3 \mathrm{p}-3 \mathrm{~s} .4 \mathrm{~s}$ & $3-3$ \\
\hline & 518.36043 & $5.75 \mathrm{e}-01$ & $2.716640-5.107828$ & $3 \mathrm{~s} .3 \mathrm{p}-3 \mathrm{~s} .4 \mathrm{~s}$ & $5-3$ \\
\hline $557[\mathrm{O} \mathrm{I}]$ & 557.7339 & $1.26 \mathrm{e}-08$ & $1.967364-4.189747$ & $2 \mathrm{~s} 2.2 \mathrm{p} 4-2 \mathrm{~s} 2.2 \mathrm{p} 4$ & $5-1$ \\
\hline \multirow{2}{*}{$\begin{array}{l}589 \mathrm{Na} \mathrm{I} \\
\text { (doublet) }\end{array}$} & 588.9950 & $6.22 \mathrm{e}-01$ & $0.000000-2.104430$ & $3 s-3 p$ & $2-4$ \\
\hline & 589.5924 & $6.18 \mathrm{e}-01$ & $0.000000-2.102298$ & $3 s-3 p$ & $2-2$ \\
\hline
\end{tabular}

${ }^{1}$ Indicates the detected lines in Fig. 3. 


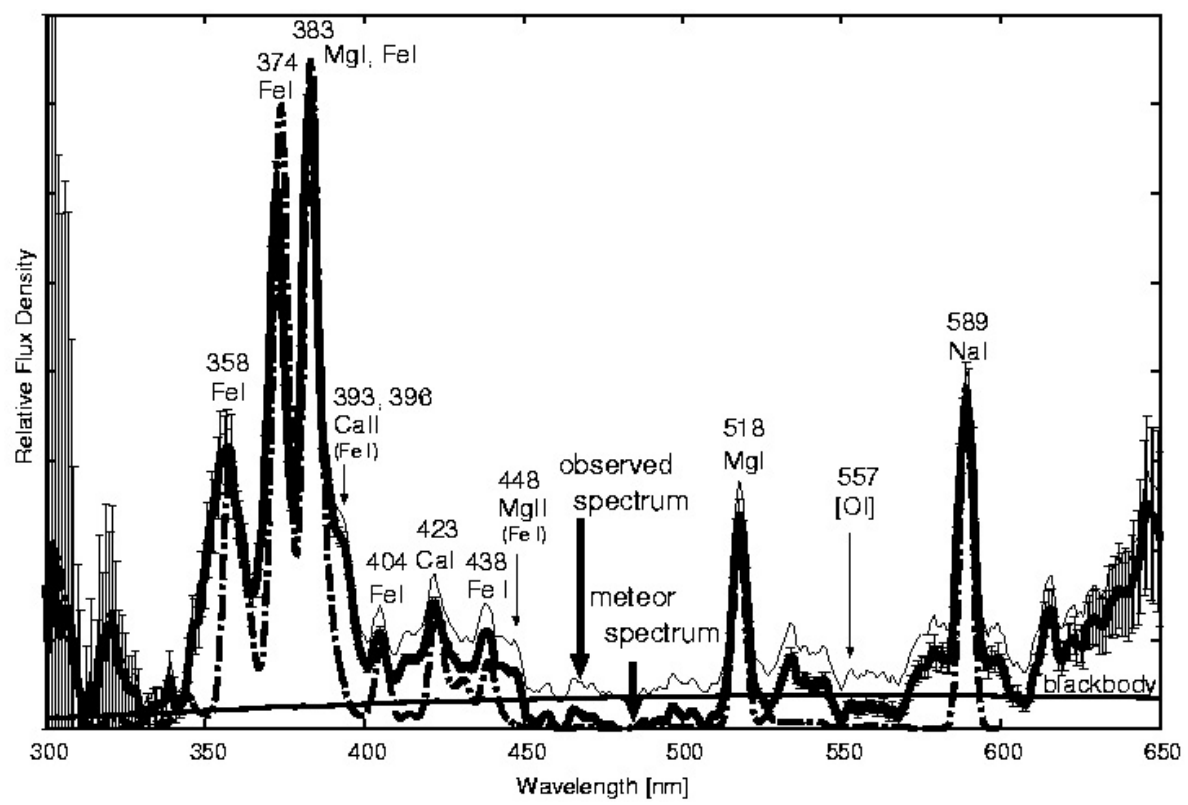

Fig. 3. An example of the reduced spectrum (narrow line) at $0.165 \mathrm{~s}$. The meteor spectrum was obtained by subtracting the blackbody radiation from the observed spectrum. Dotted lines are the best fit models by neutral atomic emissions of 358, 374, 383, 404, 423, 438, 518 and 589 nm. The excitation temperature of this frame is $5500 \mathrm{~K}$ and the blackbody temperature is $5250 \pm 250 \mathrm{~K}$.

Table 2. The observed flux ratios of 358, 374, 383, 404, 423, 438 and 589 to the $518 \mathrm{~nm}$ lines and measured line positions at $t=0.165 \mathrm{~s}$.

\begin{tabular}{ccccccccc}
\hline \hline Line position & 358 & 374 & 383 & 404 & 423 & 438 & 518 & 589 \\
\hline Flux ratio to the $518 \mathrm{~nm}$ & $3.3 \pm 0.6$ & $3.4 \pm 0.3$ & $4.4 \pm 0.4$ & $0.44 \pm 0.04$ & $0.58 \pm 0.05$ & $0.47 \pm 0.03$ & $1.0 \pm 0.1$ & $1.7 \pm 0.3$ \\
\hline
\end{tabular}

where $N_{0}$ is the total number of neutral atoms in the ground state energy level; $g_{\mathrm{u}}$ and $g_{0}$ are the statistical weights of the upper and ground state energy levels, respectively; $T_{\mathrm{e}}$ is the electronic excitation temperature; and $k_{\mathrm{B}}$ is the Boltzmann's constant. The $g$-value of each energy level is taken from the Physical Reference Data of National Institute of Standard and Technology (NIST). The flux $\widetilde{f}(\lambda)$ of a line emitted by atoms at a transition from a state u in the upper energy level $E_{\mathrm{u}}$ to a state 1 in the lower energy level $E_{1}$ is expressed as

$\widetilde{f}(\lambda)=\frac{N_{0} h c v_{\mathrm{ul}}}{4 \pi r^{2}} A_{\mathrm{ul}} \frac{g_{\mathrm{u}}}{g_{0}} \exp \left(-\frac{E_{\mathrm{u}}}{k_{\mathrm{B}} T_{\mathrm{e}}}\right) \cdot G(\lambda)$,

where $A_{\mathrm{ul}}$ is the Einstein transition probability of spontaneous emission, $v_{\mathrm{ul}}=\left(E_{\mathrm{u}}-E_{1}\right) / h c$ the wavenumber of the line, and $r$ the distance from the meteor to the observer. The spectral profile is assumed to be Gaussian, defined as

$G(\lambda)=\exp \left(\frac{-\left(\lambda-\lambda_{0}\right)^{2}}{2 \sigma^{2}}\right)$

where $\lambda_{0}$ is the wavelength of the line center, and $\sqrt{2} \sigma$ the width of the instrumental profile. In this analysis, the instrumental profile was roughly estimated from the width of the observed $423 \mathrm{~nm}$ (CaI) line, which suffers the least contamination effect of all observed lines. Then, Eq. (5) is rewritten as

$F=\frac{N_{0} h c v_{\mathrm{ul}}}{4 \pi r^{2}} A_{\mathrm{ki}} \frac{g_{\mathrm{u}}}{g_{0}} \exp \left(-\frac{E_{\mathrm{u}}}{k_{\mathrm{B}} T_{\mathrm{e}}}\right) \cdot \sqrt{2 \pi} \sigma$.
We take the line flux of $\mathrm{MgI}$ (518nm), one of the brightest lines observed in the Leonid spectra, as a standard and then evaluate the abundance ratios of $\mathrm{FeI} / \mathrm{MgI}, \mathrm{CaI} / \mathrm{MgI}$, and $\mathrm{NaI} / \mathrm{MgI}$ on the ground state level from the ratios between observed fluxes of these emission lines. It should be noted that atoms of different ionization states are regarded as independent species here. Using Eq. (7), the abundance ratio of the neutral metallic atom $X$ I relative to $\mathrm{MgI} N_{X \mathrm{I}} / N_{\mathrm{MgI}}$ at the ground state level is evaluated for each spectrum in Fig. 2. Then the observed flux $F_{X \mathrm{I}}^{(n)}$ of the $n$th line emitted by the atoms $X \mathrm{I}$ is expressed as

$\left(\frac{F_{X \mathrm{I}}^{(n)}}{F_{\mathrm{MgI}}}\right)_{\mathrm{cal}}=\frac{N_{X \mathrm{I}} v_{X \mathrm{I}}^{(n)} A_{X \mathrm{I}}^{(n)}}{N_{\mathrm{MgI}} v_{\mathrm{MgI}} A_{\mathrm{MgI}}} \frac{g_{X \mathrm{Iu}}^{(n)}}{g_{X \mathrm{I} 0}^{(n)}} \frac{g_{\mathrm{MgI} 0}}{g_{\mathrm{MgIu}}} \exp \left[-\frac{E_{X \mathrm{Iu}}^{(\mathrm{n})}-E_{\mathrm{MgI}}}{k_{\mathrm{B}} T_{\mathrm{e}}}\right]$.

The abundance ratio $N_{X \mathrm{I}} / N_{\mathrm{MgI}}$ and excitation temperature $T_{\mathrm{e}}$ are determined by the least square method so as to minimize $\Delta$, defined as

$\Delta \equiv \sum_{n}\left[\left(\frac{F_{X \mathrm{I}}^{(n)}}{F_{\mathrm{MgI}}}\right)_{\mathrm{obs}}-\left(\frac{F_{X \mathrm{I}}^{(n)}}{F_{\mathrm{MgI}}}\right)_{\mathrm{cal}}\right]^{2}=$ minimum.

With varying $N_{X \mathrm{I}} / N_{\mathrm{MgI}}$ and $T_{\mathrm{e}}$, the summation over $n$ is taken for the observed lines at 358, 374, 383, 404, 423, 438, and $589 \mathrm{~nm}$. The numbers of atoms in the ground state and all excited levels were summed to obtain the total abundance of MgI. This process resulted in the successful derivation of total neutral metallic abundance ratios. Here, we assume that $T_{\mathrm{e}}$ is common for all neutral metallic species because of the assumption 
of LTE. In reality, the observed $n$th "line" is composed of several individual atomic lines, which cannot be resolved due to low spectral resolution in the observations. Thus, $\left(F_{X \mathrm{I}}^{(n)} / F_{\mathrm{MgI}}\right)_{\mathrm{cal}}$ in Eq. (9) is the sum of fluxes from individual atomic lines (see Table 1). The ratio $\left(F_{X \mathrm{I}}^{(n)} / F_{\mathrm{MgI}}\right)_{\mathrm{obs}}$ is evaluated as the observed flux at the wavelength of the $n$th "line" ratio to the $518 \mathrm{~nm}$ (MgI) (see e.g. Table 2). The flux ratio errors were estimated by error propagation of the system efficiency curve and calibrated flux. An example of the final results is shown as a dotted line in Fig. 3.

\subsection{Consideration of ionized atom}

The procedure described above yielded excitation temperature $T_{\mathrm{e}}$, blackbody temperature $\widetilde{T}_{\mathrm{e}}$, and total number ratio relative to $\mathrm{Mg} \mathrm{I}, N_{X} / N_{\mathrm{MgI}}$ for four neutral atoms. Here, we have to consider the degree of ionization of atoms in order to obtain the elemental abundances.

In the case of this fireball, MgII (448 nm) emissions seem to have been observed in all frames in Fig. 2. This particular line is sometimes observed in a later stage for Leonids. Presence of the high temperature component is demonstrated by $\mathrm{MgII}$ (448 nm) line, which situation corresponds to the hot component condition originally proposed by Borovička (1993), introduced as follows. The meteor plasma theory is too complicated to explain all excited and ionized metallic emissions. There are a few lines visible in the spectrum, which should be infinitesimally faint (Borovička 1993). Bronsthten (1981) regarded this phenomenon as a puzzle, while Nagasawa (1978) and Jenniskens et al. (2002c) speculated about the non - LTE process. Borovička and Jenniskens (2000) assumed two types of spectra; one is "the main component" composed of neutral atomic lines and CaII lines, and the other is "the second component" composed of CaII, MgII, SiII, HI, FeII, and CrII lines. The excitation temperature of the main component is $5000 \mathrm{~K}$ and that of the second component is about $10000 \mathrm{~K}$. Borovička (1993) considers that the hotter component is caused by a meteor shock wave. We try to apply their idea in order to consider the total metallic abundances.

For the total number of atomic species $X, N_{X_{\text {total }}}$ is expressed as

$N_{X_{\text {total }}}=N_{X \text { I }}+N_{X \text { II }}$

where $N_{X \text { I }}$ is the number of the neutral atoms and $N_{X \text { II }}$ the number of the singly ionized atoms. The number of more highly ionized atoms is thought to be negligible (e.g., Borovička 1993; Borovička et al. 1996, 1999; Borovička \& Jenniskens 2000).

The ratios of the number of singly ionized atoms and of neutral atoms are given by the Saha equation expressed as

$\frac{N_{X \text { II }}}{N_{X \mathrm{I}}} \cdot n_{\mathrm{e}}=2 \cdot \frac{Z(\mathrm{II})}{Z(\mathrm{I})} \cdot \frac{\left(2 \pi m_{\mathrm{e}} k_{\mathrm{B}} T_{\text {ion }}\right)^{3 / 2}}{h^{3}} \exp \left[-\frac{\chi_{\mathrm{x}}}{k_{\mathrm{B}} T_{\text {ion }}}\right]$,

where $m_{\mathrm{e}}$ and $n_{\mathrm{e}}$ are the electron mass; the number density, $Z(\mathrm{I})$ and $Z(\mathrm{II})$, are the partition functions of the species $X \mathrm{I}$ and $X$ II; $T_{\text {ion }}$ the ionization temperature; and $\chi_{X}$ the first ionization potential (Allen 1999).

In this analysis, a value of the total metallic abundance, $\mathrm{Ca} / \mathrm{Mg}$ of the main component, is equal to the ratio of that of the hot component for the definition (Borovička 1993). The relation of the Saha's functions in the main and hot components is described as

$f_{\text {Main }}\left(\frac{N_{\mathrm{CaI}}}{N_{\mathrm{MgI}}}, T_{\text {Main }}, n_{\mathrm{e}}^{\mathrm{Main}}\right)=f_{\mathrm{Hot}}\left(\frac{N_{\mathrm{CaII}}}{N_{\mathrm{MgII}}}, T_{\mathrm{Hot}}, n_{\mathrm{e}}^{\mathrm{Hot}}\right)$,

where $f_{\text {Main }}$ and $f_{\text {Hot }}$ are Saha's functions, which is defined in the Appendix; $N_{\mathrm{CaI}} / N_{\mathrm{MgI}}$ the abundance ratio of the neutral atom on the ground state level of the main component; $N_{\text {CaII }} / N_{\text {MgII }}$ the abundance ratio of the ionized atom on the ground state level of the hot component; $T_{\text {Main }}$ the excitation temperature of the main component; $T_{\text {Hot }}$ the excitation temperature of the hot component; $n_{\mathrm{e}}^{\text {Main }}$ the electron density of the main component; and $n_{\mathrm{e}}^{\text {Hot }}$ the electron density of the hot component. From now on, the ionization temperatures $\left(=T_{\text {ion }}\right)$ for hot and main components are treated as the same value $T_{\text {Main }}$ and $T_{\text {Hot }}$. Electron density can be derived by using the relation described in the Appendix.

The $n_{\mathrm{e}}^{\text {Hot }}$ can be rewritten as $n_{\mathrm{e}}^{\text {Main }} \cdot T_{\text {Main }} / T_{\text {Hot }}$ by using the relation from the pressure of the radiant gas between the main component and hot component, which are described as $p=n_{\mathrm{e}}^{\text {Main }} k_{\mathrm{B}} T_{\text {Main }}=n_{\mathrm{e}}^{\text {Hot }} k_{\mathrm{B}} T_{\text {Hot }}$. Summation of number to the excited level abundance; $\left(N_{\mathrm{CaII}} / N_{\mathrm{MgII}}\right)_{\text {sum }}$ for time series is obtained by the method described in Kasuga et al. (2004). We take the flux of the MgII line as a standard and evaluate the abundance ratios of $\mathrm{CaII} / \mathrm{MgII}$ at ground state level from the ratio between observed fluxes of these emissions. Then, the results were obtained as listed in Table 3 and the physical parameters of the CaII $(393,396 \mathrm{~nm})$ and $\mathrm{MgII}(448 \mathrm{~nm})$ are listed in Table 1.

The analysis process under the hot component condition, as outlined above, was applied to the fireball data ranging from 0.231 to $0.330 \mathrm{~s}$ in Fig. 2. In these frames, two types of electron densities were obtained as solutions, one with a positive value, the other negative. The proper electron density is finally selected as a positive value for the definition (Borovička 1993), and the results described in Fig. 4

Even though, the application of the analysis process failed at the early stage from 0.066 to $0.198 \mathrm{~s}$, temperature of the hot component could be fitted at $10000 \mathrm{~K}$. Both derived values of electron density resulted in a negative, which is an unrealistic situation caused by low $N_{\text {CaII }} / N_{\text {MgII }}$. Usually, CaII (393, $396 \mathrm{~nm})$ emissions are much brighter than $\mathrm{MgII}(448 \mathrm{~nm})$ emissions under the hot component condition (Borovička \& Jenniskens 2000; Borovička 1993). However, in the case of this meteor, the data from 0.066 to $0.198 \mathrm{~s}$ CaII emissions are fainter than the MgII $(448 \mathrm{~nm})$. In this early stage, CaII (393, $396 \mathrm{~nm}$ ) may not be the hot component.

Here we assume that CaII was under the main component condition instead of the hot one, in order to derive electron density for the early stage. The validity of this assumption will be described later in Subsection 4.2. From 0.066 to 0.198 s frames, we assume that only the $\mathrm{MgII}(448 \mathrm{~nm})$ is under the hot component condition.

Electron density is obtained by the ratio $\left(N_{\mathrm{CaII}} / N_{\mathrm{CaI}}\right)_{\text {sum }}$ using CaII $(393,396 \mathrm{~nm})$ and $\mathrm{CaI}(423 \mathrm{~nm})$. We take the CaI line flux as a standard and evaluate the abundance ratios of CaII/CaI at ground state level from the ratio between observed fluxes of 


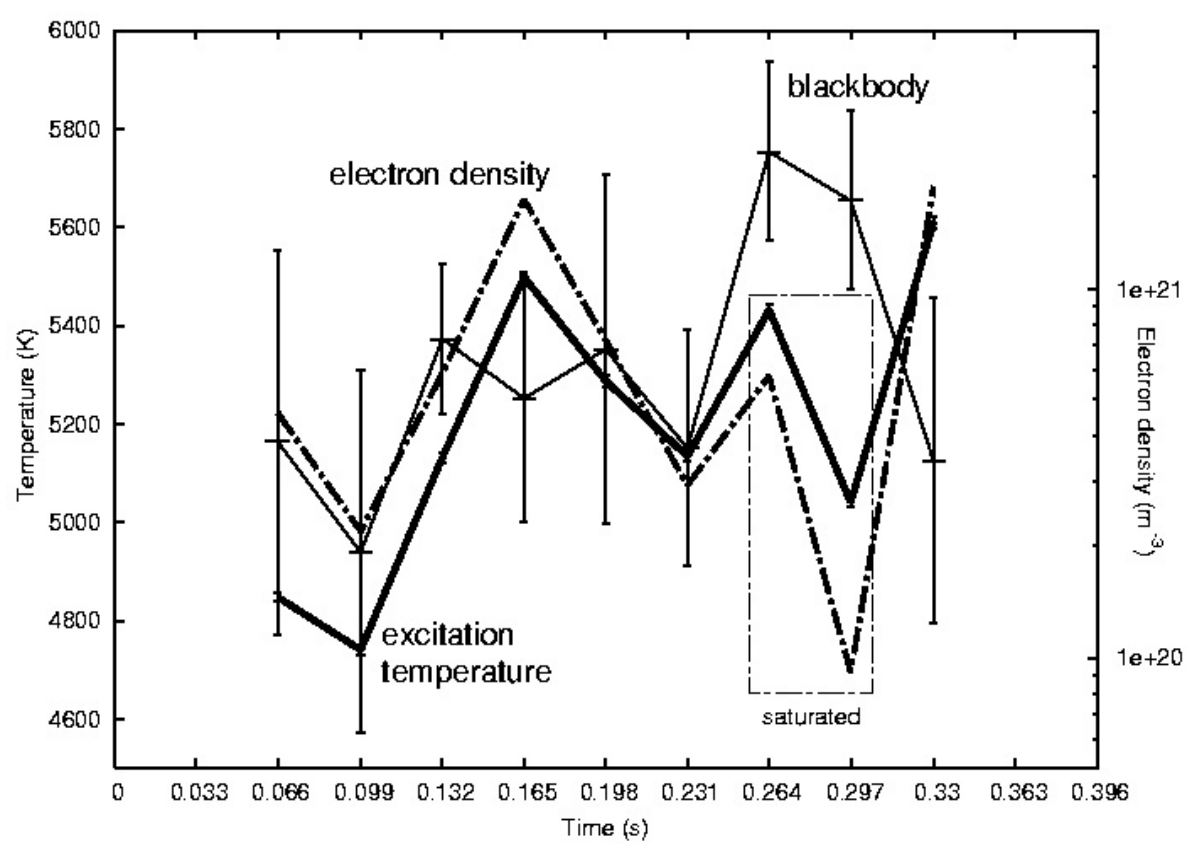

Fig. 4. Time variations of the excitation temperature (thick line), blackbody temperature (thin line) and electron density (dotted line). The $374 \mathrm{~nm}(\mathrm{FeI})$ and $383 \mathrm{~nm}(\mathrm{MgI}, \mathrm{FeI})$ lines were saturated at $t=0.264 \mathrm{~s}$ and $t=0.297 \mathrm{~s}$ so that the derived exciting temperatures cannot be used for interpretation.

Table 3. The results of the summed to the excited level abundance ratios of CaI/MgI, CaII/MgII and CaII/CaI.

\begin{tabular}{cccccccccc}
\hline \hline$t(\mathrm{~s})$ & 0.066 & 0.099 & 0.132 & 0.165 & 0.198 & 0.231 & 0.264 & 0.297 & 0.330 \\
\hline$\left(N_{\text {CaI }} / N_{\mathrm{MgI}}\right)_{\text {sum }}$ & 0.0024 & 0.0018 & 0.0023 & 0.0035 & 0.0037 & 0.0028 & 0.0058 & 0.0036 & 0.0057 \\
$\left(N_{\text {CaII }} / N_{\text {MgII }}\right)_{\text {sum }}$ & $0.00052^{*}$ & $0.00010^{*}$ & $0.0015^{*}$ & $0.0012^{*}$ & $0.0030^{*}$ & 0.027 & 0.057 & 0.059 & 0.035 \\
$\left(N_{\text {CaII }} / N_{\text {CaI }}\right)_{\text {sum }}$ & 3.6 & 5.0 & 6.6 & 6.3 & 8.5 & - & - & - & - \\
\hline
\end{tabular}

* These values are regarded as unrealistic; see text for detail.

these emissions. The obtained results are also included in both Table 3 and Fig. 4.

\section{Results and discussion}

\subsection{Abundances, temperature and electron density}

The final results are shown in Figs. 5 and 6. Figure 5 shows time variation for abundances of the metallic elements $\mathrm{Fe}, \mathrm{Ca}$, and $\mathrm{Na}$, together with their solar abundance described as horizontal lines (Anders \& Grevesse 1989). The data for $t=0.033$ and $0.363 \mathrm{~s}$ were omitted because the emission lines of the former were affected by the low signal-to-noise ratio and the latter data did not include the line $589 \mathrm{~nm}(\mathrm{NaI})$, which was not in the FOV at that time. In order to assess the accuracy of this result, we also show the relative abundance of $\mathrm{Mg}$, which must by definition be unity at any time. It is, in fact, close to unity at all times, which supports the validity of the LTE assumption in this study.

One of the remarkable features in Fig. 5 is that the abundance ratio of $\mathrm{Fe} / \mathrm{Mg}$ is always lower than the solar abundance. The average value, except for the saturated period, is $(\mathrm{Fe} / \mathrm{Mg}) /(\mathrm{Fe} / \mathrm{Mg})_{\text {solar }}=0.61$, as shown in Table 4 . This might indicate the presence of a $\mathrm{Mg}$-rich silicate meteoroid as observed in comets, for example Hale-Bopp (Wooden et al. 2000). $\mathrm{Ca} / \mathrm{Mg}$ is also always lower than the solar abundance ratio, $(\mathrm{Ca} / \mathrm{Mg}) /(\mathrm{Ca} / \mathrm{Mg})_{\text {solar }}=0.28$, as shown in Table 4. There are two possibilities: one is the refractoriness of $\mathrm{Ca}$, which will be discussed later. Another possibility is the intrinsic depletion of $\mathrm{Ca}$ as seen in some interplanetary dust particles (IDPs) (Arndt et al. 1996). Na/Mg has a value similar to that of the solar abundance, and the average value is $(\mathrm{Na} / \mathrm{Mg}) /(\mathrm{Na} / \mathrm{Mg})_{\text {solar }}=1.1$ except for the saturated period, as shown in Table 4. Recent work by Trigo-Rodríguez et al. (2003, 2004) shows that the averaged metallic abundances of Leonid meteors almost agree with solar abundances except for $\mathrm{Ca}$ and $\mathrm{Na}$; while $\mathrm{Ca}$ abundance is lower than solar abundance, $\mathrm{Na}$ is more abundant than the solar. Our result for the averaged $\mathrm{Na}$ abundance also shows it to be slightly higher than the solar abundances, which is consistent with their conclusion.

Time variations of excitation temperature $T_{\mathrm{e}}$ and the blackbody temperature are shown in Fig. 4. Excitation temperature roughly agrees with $T_{\mathrm{e}}$ of the Leonids estimated by Trigo-Rodríguez et al. (2003). The Leonids show the highest excitation temperature because of the highest kinetic energy caused by the retrograde trajectory (Trigo-Rodríguez et al. 2003). The excitation temperature value of June Boötid 


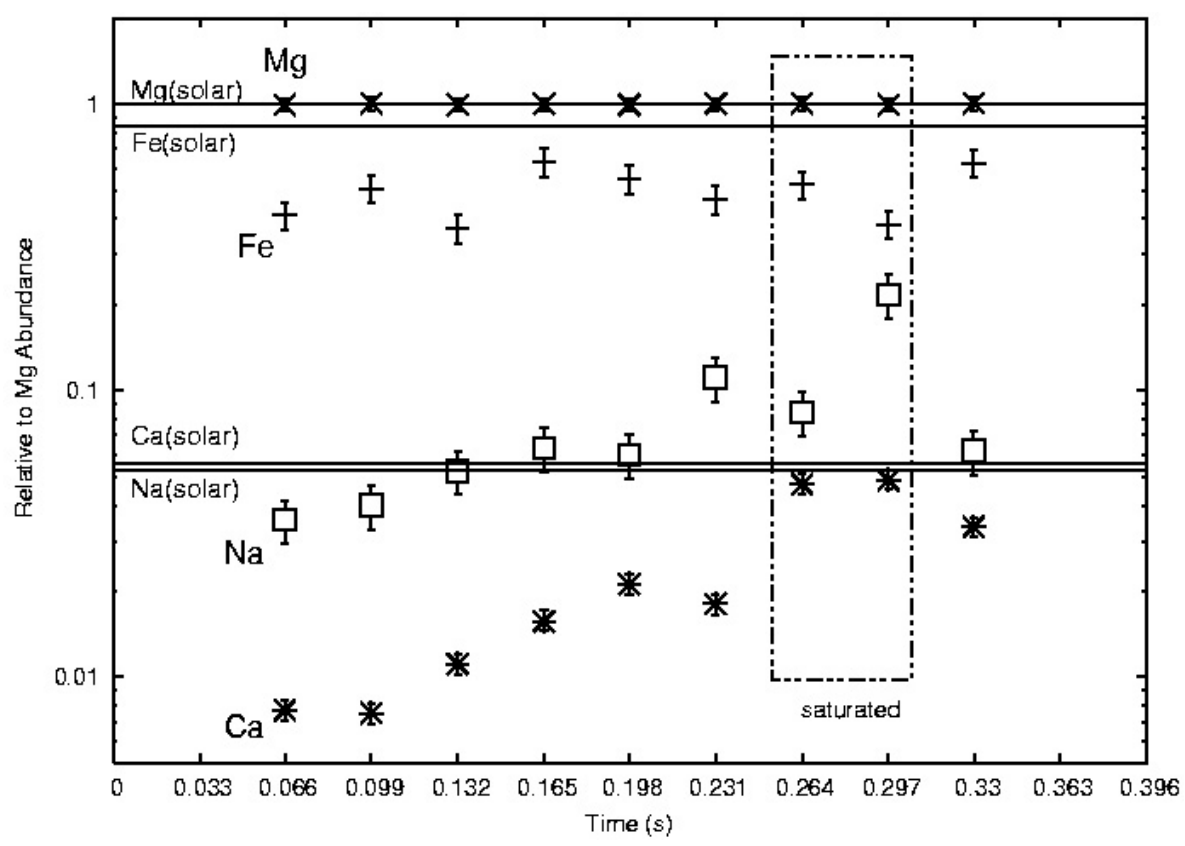

Fig. 5. Time variation of the abundances of $\mathrm{Mg}, \mathrm{Fe}, \mathrm{Ca}$ and Na relative to $\mathrm{Mg}$. The solar abundances (Anders \& $\mathrm{Grevesse} 1989$ ) of $\mathrm{Fe}, \mathrm{Ca}, \mathrm{Na}$ and $\mathrm{Mg}$ are shown by the horizontal lines. The $374 \mathrm{~nm}$ (FeI) and $383 \mathrm{~nm}$ (MgI, FeI) lines were saturated by the strong meteor emissions at $t=0.264 \mathrm{~s}$ and $t=0.297 \mathrm{~s}$.

Table 4. Comparison of the neutral abundances with the total abundances and singly ionization energies for each element.

\begin{tabular}{cccc}
\hline \hline & $\mathrm{Fe} / \mathrm{Mg}$ & $\mathrm{Ca} / \mathrm{Mg}$ & $\mathrm{Na} / \mathrm{Mg}$ \\
\hline Neutral & $0.58 \pm 0.07$ & $0.0027 \pm 0.0002$ & $0.0048 \pm 0.0008$ \\
Total (neutral + ion) & $0.51 \pm 0.06$ & $0.016 \pm 0.001$ & $0.059 \pm 0.01$ \\
Solar (Anders \& Grevesse 1989) & 0.84 & 0.057 & 0.054 \\
Ionization energy [eV] & $\chi_{\mathrm{Fe}}=7.87$ & $\chi_{\mathrm{Ca}}=6.113$ & $\chi_{\mathrm{Na}}=5.139$ \\
\hline
\end{tabular}

meteor is low, which agrees with their slow-moving velocity (Kasuga et al. 2004). One notable feature is that the values of excitation temperature and blackbody temperature exhibit similar time variation except at maximum brightness, which also supports the validity of the LTE condition.

At maximum brightness, $T_{\mathrm{e}}$ probably cannot be derived, due to saturation of the $374 \mathrm{~nm}$ (FeI) and $383 \mathrm{~nm}$ (MgI, FeI) lines. At the end of the brightness at $t=0.330 \mathrm{~s}, T_{\mathrm{e}}$ does not coincide with blackbody temperature. This situation may indicate a non-LTE condition at this final stage of the meteor. The several local peaks in the excitation and blackbody temperatures may suggest inhomogeneous structure for the ablated meteoroids.

Figure 6 shows the relationship between excitation temperature and abundances of the metallic elements. Several data plotted in this figure should be carefully interpreted, including saturated data and data from the early phase, as will be discussed in the next subsection. This figure indicates that the abundance of $\mathrm{Ca}$ increases with excitation temperature, although there is no clear trend in the Fe and $\mathrm{Na}$.

This correlation depends on the volatility of these elements during meteoroid ablation. It should be noted that $\mathrm{Ca}$ is more refractory than $\mathrm{Mg}, \mathrm{Fe}$, and $\mathrm{Na}$ (e.g., Grossman 1974; Field 1974). The reasons for the absence of trends in $\mathrm{Fe}$ and $\mathrm{Na}$ in Fig. 6 may be explained by how close Fe and $\mathrm{Na}$ abundances are to solar abundances at any excitation temperature in Fig. 6. This indicates that $\mathrm{Fe}$ and $\mathrm{Na}$ were evaporated completely at the beginning of emission. These features may be caused by the Leonid meteoroids' high speed.

$\mathrm{Ca}$ is, however, a more refractory element than the others, as stated above. Ca can be easily affected by incomplete evaporation among these elements, as described in Trigo-Rodríguez et al. (2003) and Borovička et al. (1999). The Ca trend in the correlation between excitation temperature and abundance shows the evaporation process. Even at the highest excitation temperature, $\mathrm{Ca}$ abundance is lower than the solar value (Anders \& Grevesse 1989), which is consistent with previous research (e.g. Trigo-Rodríguez et al. 2003; Borovička et al. 1999). Although the intrinsic depletion of $\mathrm{Ca}$ abundance relative to solar value may be possible, the presence of correlation in Fig. 6 strongly suggests incomplete evaporation of Ca due to refractoriness.

Here, we note error estimates for the derived physical parameters in the above discussion. Errors for metallic abundances are estimated by the errors in both system efficiency and flux in Figs. 1 and 3. Errors of fitted excitation temperatures are not included, because they are unrealistically small, while errors of metallic abundances correspond to several kelvin (K) 


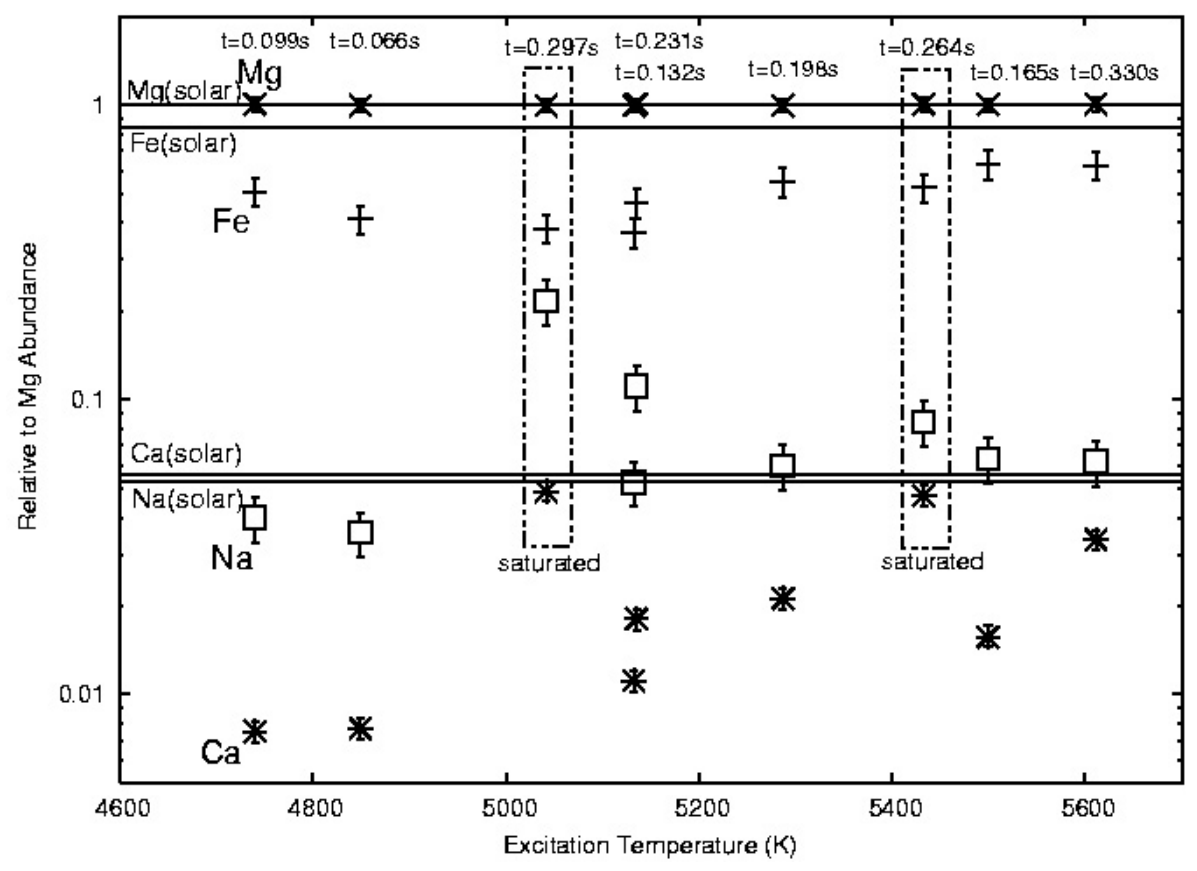

Fig. 6. The abundances of the metallic elements $\mathrm{Fe}, \mathrm{Ca}$ and $\mathrm{Na}$ relative to $\mathrm{Mg}$ vs. the excitation temperature.

for excitation temperatures. Errors in the least square method are also described in Borovička (1993); the formal standard deviations from the least squares method are small, e.g. less than $10 \mathrm{~K}$ for temperature.

\subsection{Early emission phase}

The fast-moving meteor plasma mechanism is very complicated, so that previous papers (e.g. Borovička 1993; Jenniskens et al. 2002c) have assumed the hot component condition thought to be caused by the shock wave. We also applied this idea here, as mentioned in Sect. 3.3. However, in the case of this meteor, $\mathrm{CaII}(393,396 \mathrm{~nm})$ and $\mathrm{MgII}(448 \mathrm{~nm})$ could not be explained as hot components during the early emission phase $(t=0.066-0.198 \mathrm{~s})$ in Fig. 2, although they could be explained in terms of a hot component condition during the latter emission phase ( $t=0.231-0.330 \mathrm{~s})$. In order to explain CaII as the hot component during the early emission phase, CaII abundance has to be a very low value below the excitation temperature of $10000 \mathrm{~K}$. In this condition, derived electron densities show unrealistically negative values, thus contradicting the hot component theory (Borovička 1993).

There are two possible explanations for this situation. One is the idea that CaII belongs to the main component, not to the hot component in the early phase. We applied this idea in order to derive electron density as a trial in Sect. 3.3. Another possibility is that observed emissions in the early phase near $448 \mathrm{~nm}$ are caused by neutral atoms such as FeI. We might misidentify these emissions of neutral atoms as ionized lines, due to their faintness and low spectral resolution. If this is the case, derived values of the abundance ratios would be meaningless, at least in the early phase. It should be emphasized that the derived values in the early phase shown in this study are based on the former assumption, which is on the idea that CaII belongs to the main component. At this stage, we cannot determine which of these two ideas is more likely. In the future, a trial is needed to clarify circumstances in the early phase, something beyond the scope of this paper.

It should be emphasized that this is one of very few clear demonstrations of time variations in the physical parameters of Leonid meteors along their trajectories. More samples of high resolution spectra will definitely be needed in further discussions of this early emission phase.

\section{Conclusions}

We carried out HDTV spectroscopic observations of the 2002 Leonid meteor shower on the Leonid MAC 2002 mission, and analyzed one representative meteor spectrum obtained at the $03^{\mathrm{h}} 47^{\mathrm{m}} 54^{\mathrm{s}}$ UT, November 19,2002 . From analysis of this spectrum, we obtained the time variation of the abundances of metallic atoms, the electronic excitation temperature, the blackbody temperature and electron density. It is found that the metallic abundances in this Leonid meteor under the LTE condition differ slightly from solar abundances. The Ca abundance, which shows time variation along the trajectory, seems to correlate with the temperatures. These results indicate that the spectrum data in high time resolution by the HDTV system enabled us to clarify the time variation affected by meteoroid dust compositions and their volatility.

We have reached the following conclusions about this meteoroids:

1. Metallic abundances of $\mathrm{Fe}$ and $\mathrm{Ca}$ relative to $\mathrm{Mg}$ are slightly different from their solar abundances.

2. Na abundance is instead higher than solar abundance in averaged value. 
3. Mg-rich abundance to $\mathrm{Fe}$ is consistent with the existence of $\mathrm{Mg}$-Rich silicate in comets.

4. A correlation was found between the excitation temperature and $\mathrm{Ca}$ abundance.

5. It is likely that Ca could not be completely evaporated during the meteor ablation, rather than $\mathrm{Ca}$ depleting intrinsically in the meteoroids.

6. The search for bands of interesting molecules, such as $\mathrm{OH}$ and $\mathrm{CN}$, were not successful in this study.

Acknowledgements. The authors are grateful to Dr. Peter Jenniskens for organizing the Leonid MAC 2002 mission. The mission was successful with the support of the NASA Ames Research Center (USA), Edwards Air Force Base (USA), Offutt Air Force Base (USA), Torrejon Air Force Base (Spain), and the Center for Astrobiology (Spain). The Leonid project in Japan was supported by grants from the National Observatory of Japan (NAOJ) and the Japan Space Forum (JSF). T.K. is thankful to Drs. A. Fujiwara, M. Abe, S. Hasegawa, M. Ishiguro (ISAS/JAXA), Y. Hirahara, S. Watanabe, S. Sirono, S. Yoshida and T. Ootsubo (Nagoya Univ.) for their kind support of this work. T.Y. acknowledges the support of a grant from the Institute of Low Temperature Science, Hokkaido University and grants-in-aid from the Japan Society for the Promotion of Science.

\section{Appendix A}

We show how to derive the electron density in the Appendix. We define Saha's functions as

$f_{\text {Main }}\left(\frac{N_{\mathrm{CaI}}}{N_{\mathrm{MgI}}}, T_{\text {Main }}, n_{\mathrm{e}}^{\mathrm{Main}}\right)=\frac{N_{\mathrm{CaI}}^{\mathrm{Main}}+N_{\mathrm{Ca} \text { II }}^{\mathrm{Main}}}{N_{\mathrm{Mg} \mathrm{I}}^{\mathrm{Main}}+N_{\mathrm{Mg} \text { II }}^{\text {Main }}}$

$f_{\text {Hot }}\left(\frac{N_{\text {CaII }}}{N_{\mathrm{MgII}}}, T_{\mathrm{Hot}}, n_{\mathrm{e}}^{\mathrm{Hot}}\right)=\frac{N_{\mathrm{CaI}}^{\mathrm{Hot}}+N_{\mathrm{CaII}}^{\mathrm{Hot}}}{N_{\mathrm{MgI}}^{\mathrm{Hot}}+N_{\mathrm{Mg} \text { II }}^{\mathrm{Hot}}}$,

where $N_{\mathrm{Mg} \text { I }}^{\mathrm{Main}}$ is $\mathrm{Mg}$ I abundance summed to excited level in the main component, $N_{\mathrm{Mg} \text { II }}^{\mathrm{Main}}$ is $\mathrm{Mg}$ II abundance summed to excited level in the main component, $N_{\mathrm{CaI}}^{\mathrm{Main}}$ is $\mathrm{Ca}$ I abundance summed to excited level in the main component, $N_{\mathrm{Ca} \text { II }}^{\mathrm{Main}}$ is $\mathrm{Ca}$ II abundance summed to excited level in the main component, $N_{\mathrm{MgI}}^{\mathrm{Hot}}$ is $\mathrm{MgI}$ abundance summed to excited level in the hot component, $N_{\mathrm{Mg} \text { II }}^{\mathrm{Hot}}$ is $\mathrm{Mg}$ II abundance summed to excited level in the hot component, $N_{\mathrm{CaI}}^{\mathrm{Hot}}$ is $\mathrm{Ca}$ I abundance summed to excited level in the hot component and $N_{\mathrm{Ca} \text { II }}^{\mathrm{Hot}}$ is Ca II abundance summed to excited level in the hot component. From Saha's equation, we can derive the number of ionized species from the main component as

$$
\begin{aligned}
N_{\mathrm{Mg} \mathrm{II}}^{\mathrm{Main}} & =\frac{N_{\mathrm{Mg} \mathrm{I}}^{\text {Main }}}{n_{\mathrm{e}}^{\text {Main }}} \cdot 2 \cdot \frac{Z(\mathrm{Mg} \mathrm{II})}{Z(\mathrm{Mg} \mathrm{I})} \cdot \frac{\left(2 \pi m_{\mathrm{e}} k_{\mathrm{B}} T_{\text {Main }}\right)^{3 / 2}}{h^{3}} \exp \left[-\frac{\chi_{\mathrm{Mg}}}{k_{\mathrm{B}} T_{\text {Main }}}\right] \\
& =\frac{N_{\text {Mg I }}^{\text {Main }}}{n_{\mathrm{e}}^{\text {Main }}} \cdot s\left(T_{\text {Main }}, \mathrm{Mg}\right), \\
N_{\text {Ca II }}^{\text {Main }} & =\frac{N_{\text {Ca I }}^{\text {Main }}}{n_{\mathrm{e}}^{\text {Main }}} \cdot 2 \cdot \frac{Z(\mathrm{Ca} \mathrm{II})}{Z(\mathrm{Ca} \mathrm{I})} \cdot \frac{\left(2 \pi m_{\mathrm{e}} k_{\mathrm{B}} T_{\text {Main }}\right)^{3 / 2}}{h^{3}} \exp \left[-\frac{\chi_{\mathrm{Ca}}}{k_{\mathrm{B}} T_{\text {Main }}}\right] \\
& =\frac{N_{\text {Cain }}^{\text {Main }}}{n_{\mathrm{e}}^{\text {Main }}} \cdot s\left(T_{\text {Main }}, \mathrm{Ca}\right),
\end{aligned}
$$

Similarly, we can derive the number of metallic atoms for the hot component as

$$
\begin{aligned}
N_{\mathrm{MgI}}^{\mathrm{Hot}} & =\frac{N_{\mathrm{Mg} \mathrm{II}}^{\mathrm{Hot}} \cdot n_{\mathrm{e}}^{\mathrm{Hot}}}{2 \cdot \frac{Z(\mathrm{Mg} \mathrm{II})}{Z(\mathrm{Mg} \mathrm{I})} \cdot \frac{\left(2 \pi m_{\mathrm{e}} k_{\mathrm{B}} T_{\mathrm{Hot}}\right)^{3 / 2}}{h^{3}} \exp \left[-\frac{\chi_{\mathrm{Mg}}}{k_{\mathrm{B}} T_{\mathrm{Hot}}}\right]} \\
& =\frac{N_{\mathrm{Mg} \mathrm{II}}^{\mathrm{Hot}} \cdot n_{\mathrm{e}}^{\mathrm{Hot}}}{s\left(T_{\mathrm{Hot}}, \mathrm{Mg}\right)}, \\
N_{\mathrm{CaI}}^{\mathrm{Hot}} & =\frac{N_{\mathrm{Ca} \text { II }}^{\mathrm{Hot}} \cdot n_{\mathrm{e}}^{\mathrm{Hot}}}{2 \cdot \frac{Z(\mathrm{Ca} \mathrm{II})}{Z(\mathrm{CaI})} \cdot \frac{\left(2 \pi m_{\mathrm{e}} k_{\mathrm{B}} T_{\mathrm{Hot}}\right)^{3 / 2}}{h^{3}} \exp \left[-\frac{\chi_{\mathrm{Ca}}}{k_{\mathrm{B}} T_{\mathrm{Hot}}}\right]} \\
& =\frac{N_{\mathrm{Ca} \text { II }}^{\mathrm{Hot}} \cdot n_{\mathrm{e}}^{\mathrm{Hot}}}{s\left(T_{\mathrm{Hot}}, \mathrm{Ca}\right)} .
\end{aligned}
$$

The total metallic abundance; $\mathrm{Ca} / \mathrm{Mg}$ of the main component is equal to the ratio of that of hot component for the definition (Borovička 1993). Then the relation is described as:

$$
\begin{aligned}
\mathrm{Ca} / \mathrm{Mg} & =f_{\text {Main }}\left(\frac{N_{\mathrm{CaI}}}{N_{\mathrm{MgI}}}, T_{\text {Main }}, n_{\mathrm{e}}^{\mathrm{Main}}\right) \\
& =f_{\mathrm{Hot}}\left(\frac{N_{\mathrm{CaII}}}{N_{\mathrm{MgII}}}, T_{\mathrm{Hot}}, n_{\mathrm{e}}^{\mathrm{Hot}}\right)
\end{aligned}
$$

where $m_{\mathrm{e}}$ and $n_{\mathrm{e}}$ are the electron mass and the number density as stated in the body of the paper, $Z(X \mathrm{I})$ and $Z(X$ II $)$ are the partition functions of the species $X \mathrm{I}$ and $X \mathrm{II}, T_{\text {ion }}$ is the ionization temperature and $\chi_{X}$ is the first ionization potential of the species $X$ (Allen 1999). The functions $s(T, X)$ are applied using the temperature for each component and the species $X$ for simplicity.

Then we could obtain Eq. (A.8)

$$
\begin{aligned}
& n_{\mathrm{e}}^{2} \cdot\left[\frac{T_{\text {Main }}}{T_{\mathrm{Hot}}} \cdot\left(\frac{N_{\mathrm{CaI}}}{N_{\mathrm{MgI}}}\right)_{\text {sum }}^{\text {Main }}\left(\frac{N_{\mathrm{MgII}}}{N_{\mathrm{CaII}}}\right)_{\text {sum }}^{\mathrm{Hot}}\left(\frac{s\left(T_{\mathrm{Hot}}, \mathrm{Ca}\right)}{s\left(T_{\mathrm{Hot}}, \mathrm{Mg}\right)}\right)-\frac{T_{\text {Main }}}{T_{\mathrm{Hot}}}\right] \\
& +n_{\mathrm{e}} \cdot\left(s\left(T_{\text {Main }}, \mathrm{Ca}\right) \cdot \frac{T_{\text {Main }}}{T_{\text {Hot }}}+s\left(T_{\text {Hot }}, \mathrm{Mg}\right)\right) \\
& \times\left(\frac{N_{\mathrm{CaI}}}{N_{\mathrm{MgI}}}\right)_{\text {sum }}^{\text {Main }}\left(\frac{N_{\mathrm{MgII}}}{N_{\mathrm{CaII}}}\right)_{\text {sum }}^{\mathrm{Hot}}\left(\frac{s\left(T_{\mathrm{Hot}}, \mathrm{Ca}\right)}{s\left(T_{\mathrm{Hot}}, \mathrm{Mg}\right)}\right) \\
& -n_{\mathrm{e}} \cdot\left(\frac{T_{\text {Main }}}{T_{\text {Hot }}} \cdot s\left(T_{\text {Main }}, \mathrm{Mg}\right)+s\left(T_{\text {Hot }}, \mathrm{Ca}\right)\right) \\
& +s\left(T_{\mathrm{Hot}}, \mathrm{Mg}\right) \cdot s\left(T_{\mathrm{Main}}, \mathrm{Ca}\right) \cdot\left(\frac{N_{\mathrm{CaI}}}{N_{\mathrm{MgI}}}\right)_{\text {sum }}^{\text {Main }} \\
& \times\left(\frac{N_{\mathrm{MgII}}}{N_{\mathrm{CaII}}}\right)_{\text {sum }}^{\mathrm{Hot}}\left(\frac{s\left(T_{\mathrm{Hot}}, \mathrm{Ca}\right)}{s\left(T_{\mathrm{Hot}}, \mathrm{Mg}\right)}\right) \\
& -s\left(T_{\text {Hot }}, \mathrm{Ca}\right) \cdot s\left(T_{\text {Main }}, \mathrm{Mg}\right)=0
\end{aligned}
$$

where $\left(N_{\mathrm{CaI}} / N_{\mathrm{MgI}}\right)_{\text {sum }}^{\text {Main }}$ and $T_{\text {Main }}\left(=T_{\mathrm{e}}\right)$ are already yielded by the the procedure described in Sect. 3.2, and $T_{\mathrm{Hot}}$ is fixed as $10000 \mathrm{~K}$ by the previous papers (e.g. Borovička 1993) for simplicity.

\section{References}

Allen, C. W. 1999, Astronomical Quantities, Fourth ed, University of London (The Althone Press) 
Anders, E., \& Grevesse, N. 1989, Geochim. Cosmochim. Acta, 53, 197

Arndt, P., Bohsung, J., Maetz, M., et al. 1996, Meteor. Planet. Sci., 31, 817

Borovička, J. 1993, A\&A, 279, 627

Borovička, J. 1994, A\&A, 103, 83

Borovička, J., Zimnikoval, P., \& Skvarka, J. 1996, A\&A, 306, 995

Borovička, J., Stork, R., \& Bocek, J. 1999, Meteor. Planet. Sci., 34, 987

Borovička, J., \& Jenniskens, P. 2000, Earth, Moon and Plan., 82/83, 399

Bronshten, V. A. 1983, Physics of Meteoric Phenomena (Dordrecht), 1983

Field, G. B. 1974, ApJ, 187, 453

Gong, S. S., Yang, G. T., Wang, J. M., et al. 2003, Geophys. Res. Lett., 30,13

Grossman, L. 1974, Presented at Proc. of the Soviet-Am. Conf. on the Cosmochemistry of the Moon and Planets

Jenniskens, P., \& Butow, S. J. 1999, Meteor. Planet. Sci., 34, 933

Jenniskens, P., Wilson, M. A., Packan, D., et al. 2000a, Earth, Moon and Plan., 82/83, 57

Jenniskens, P., Butow, S., \& Fonda, M. 2000b, Earth, Moon and Plan., $82 / 83,1$

Jenniskens, P. 2002a, WGN (J. Internat. Meteor Org.), 30, 218

Jenniskens, P. 2002b, Proc. Asteroids, Comets, Meteors - ACM 2002. International Conference, 29 July-2 August 2002, Berlin, Germany, ed. B. Warmbein, Noordwijk, Netherlands, ESA SP500, 117
Jenniskins, P., Tedesco, E., Murthy, J., et al. 2002c, Meteor. Planet. Sci., 37, 1071

Jenniskens, P. 2003, EGS-AGU-EUG Joint Assembly, abstract No. 10288

Kasuga, T., Ebizuka, N., Abe, S., et al. 2003, The Institute of Space and Astronautical Science Rep. SP, 16, 159

Kasuga, T., Watanabe, J., Ebizuka, N., et al. 2004, A\&A, 424, L35

Karkoschka, E. 1994, Icarus, 111, 1, 174

Lyytinen, E. J., \& Van Flandern, T. 2000, Earth, Moon and Plan., $82 / 83,149$

McNaught, R. H., \& Asher, D. J. 1999, Meteor. Planet. Sci., 34, 975

McNaught, R. H., \& Asher, D. J. 2001, WGN, J. Internat. Meteor Org., 29, 156

McNaught, R. H., \& Asher, D. J. 2002, WGN (J. Internat. Meteor Org.), 30, 132

Millman, P. M., Cook, A. F., \& Hemenway C. L. 1971, Can. J. Phys., 49,1365

Nagasawa, K. 1983, Ann. Tokyo Astron. Obs. 2nd Ser., 16, 157

Trigo-Rodríguez, J. M., Llorca, J., Borovicka, J., et al. 2003, Meteor. Planet. Sci., 38, 1283

Trigo-Rodríguez, J. M., Llorca, J., Fabregat, J. 2004, MNRAS, 348, 802

Vaubaillon, J. 2002, WGN (J. Internat. Meteor Org.), 30, 144

Watanabe, J., Abe, S., Takahashi, M., et al. 1999, J. Geophys. Res., 26, 1117

Wooden, D. H., Butner, H. M., Harker, D. E., et al. 2000, Icarus, 143, 126 